$V_{T}$

NCER Working Paper Seriés

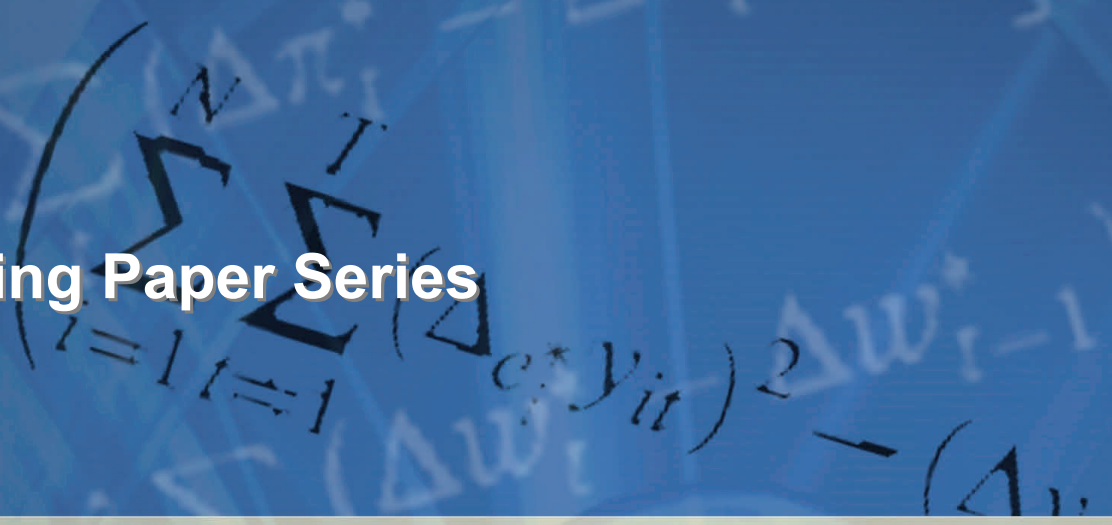

Issues in Estimating New Keynesian Phillips Curves in the Presence of Unknown Structural Change

Mariano Kulish Adrian Pagan

Working Paper \#94 October 2013 


\title{
Issues in Estimating New Keynesian Phillips Curves in the Presence of Unknown Structural Change*
}

\author{
Mariano Kulish ${ }^{\dagger}$ and Adrian Pagan ${ }^{\ddagger}$
}

September 6, 2013

\section{Contents}

1 Introduction 3

2 The Model and Estimators 5

3 A Simple Variant of the Solution Algorithm 8

4 Simulation Experiments $\quad 11$

4.1 Parameter Values . . . . . . . . . . . . . . . . . . . . . 11

4.2 The Experiments . . . . . . . . . . . . . . . . . . 12

4.2.1 Experiment 1: No breaks . . . . . . . . . . . . 12

4.2.2 Experiment 2: Breaks in Means but not in the Intercept of the Phillips Curve . . . . . . . . . . . . 14

4.2.3 Experiment $3 \pi$ Breaks, Intercept in Phillips Curve Shifts 15

4.2.4 Experiment $4 x$ Breaks, Intercept in Phillips Curve Shifts 16

*We thank Sophocles Mavroeidis and two referees for comments on an earlier draft.

†School of Economics, University of New South Wales, m.kulish@unsw.edu.au. Much of the work was done when the author was in the Research Department of the Reserve Bank of Australia

${ }^{\ddagger}$ School of Economics, University of Sydney and Melbourne Institute of Applied Economic and Social Research; adrian.pagan@sydney.edu.au 
4.2.5 Experiment 5 - Experiment 3 but Agents think Breaks at $60 \%$ of Sample . . . . . . . . . . . . 17

5 Breaks and the Euro Area New Keynesian Phillips Curve 18

6 Breaks and the U.S. New Keynesian Phillips Curve 22

$\begin{array}{lll}7 & \text { Conclusion } & 23\end{array}$

8 References $\quad 24$

9 Appendix $\quad 24$

\begin{abstract}
Many papers which have estimated models with forward looking expectations have reported that the magnitude of the coefficients of the expectations term is very large when compared with the effects coming from past dynamics. This has sometimes been regarded as implausible and led to the feeling that the expectations coefficient is biased upwards. A relatively general argument that has been advanced is that the bias could be due to structural changes in the means of the variables entering the structural equation. An alternative explanation is that the bias comes from weak instruments. In this paper we investigate the issue of upward bias in the estimated coefficients of the expectations variable based on a model where we can see what causes the breaks and how to control for them. We conclude that weak instruments are the most likely cause of any bias and note that structural change can affect the quality of instruments. We also look at some empirical work in Castle et al. (2011) on the NK Phillips curve in the Euro Area and U.S, assessing whether the smaller coefficient on expectations that Castle et al. (2011) highlight is due to structural change. Our conclusion is that it is not. Instead it comes from their addition of variables to the NKPC. After allowing for the fact that there are weak instruments in the estimated re-specified model it would seem that the forward coefficient estimate is actually quite high rather than low.
\end{abstract}




\section{Introduction}

Many papers that estimate models with forward looking expectations report that the magnitude of the coefficient attached to the forward expectations term is very large when compared with that attached to the term representing past dynamics. This has often been regarded as implausible, leading to the conjecture that the estimator of the former is biasedupwards. Why that should be the case is less clear. One possibility is that weak instruments can result in an estimator bias in small samples e.g. Mavroeidis (2004), although there is no reason to think it is upwards. Another possibility is specification error in the structural equation containing the expectations. Whilst there is little one can say about this in general, as the nature of the specification error will be crucial, a specific argument has been that the bias could be due to changes in the unconditional means of the variables entering the structural equation. Russell et al. (2010) give this explanation. Using Bai-Perron tests they find that there were 8 breaks in the unconditional mean of the U.S. inflation rate over 1960-2007. Assuming that the time of these breaks coincides with changes in the intercept of the New Keynesian Phillips curve (NKPC) they then augment the NKPC with dummy variables so as to capture intercept breaks. Re-estimating with such dummies it was found that the coefficient of the expectations term was greatly reduced. This led them to conclude that ( $\mathrm{p} 1$ ), "Once the shifts in the mean rate of inflation have been accounted for in the estimation of the United States Phillips curves we find that ... there is no significant role for expected inflation in the NK and hybrid models of inflation".

Castle et al. (2011) provide an explanation of what Russell et al. found. It revolves around the fact that a standard way of estimating an equation with forward looking expectations involves replacing the expectations term with future values of inflation and then applying an instrumental variables estimator to the resulting equation in observables. If the intercept breaks are unaccounted for when estimating the NKPC a specification error exists, and this will be correlated with future values of inflation. Thus Castle et al's (2011) argument is that the explanatory power of the future observable term is due to the breaks and not to forward-looking expectations. Technically, one gets an inconsistency in the instrumental variables (IV) estimator of the coefficient of the future expectations term. In some simple experiments they show that this effect can be substantial.

In this paper we investigate the issue of upward bias in the estimated 
coefficients of the expectations variable based on a model where we can see what causes the breaks and how to control for them. Since many of the applications involve an NKPC we work with that as the structural equation, embedding it in a simplified New Keynesian (NK) model that also has equations for real marginal cost and an interest rate rule. In each case the agent may know of the breaks but the econometrician is assumed to be ignorant of when they occur. We discuss how to solve this model in the presence of breaks, both when agents know exactly where the breaks occur and also when they get the time of the break wrong. The method of solution does not depend on the simple model we use for experiments but can be used for any model with forward looking expectations. It is set out in detail in Kulish and Pagan (2012).

Because the model is simple, we are able to perform an experiment in which there are breaks in the means of the target inflation rate and real marginal cost but in which these offset one another exactly so as to produce no breaks in the intercept of the NKPC. This experiment just makes the point that breaks in the means of variables such as inflation may not cause breaks in the intercepts of the NKPC. Yet in this experiment we find a bias in some commonly used estimators. Since the equation is correctly specified, due to the intercept being constant ( and in this experiment we assume that agents know exactly the timing of the mean shifts), the only reason that this can occur is the presence of weak instruments. This leads us to make a distinction between large-sample biases due to specification errors ( in the structural equation) and those arising in smaller samples which can come from weak instruments. The importance of the latter can sometimes be reduced, either by using different estimators or by performing inference with methods robust to weak instruments - Magnusson and Mavroeidis (2010) - while the former can't be accounted for in this way, unless the specification error can be precisely described. We find that breaks in the means of the series can often change the properties of instruments a great deal, and this may well be a bigger source of small sample bias than that coming from specification error. Moreover, we also find that the direction of the specification bias is not predictable. With some estimators and breaks it is the case that the coefficient of the expectations variable is over-estimated, while with others it is under-estimated. This leads to the conclusion that it is necessary to check for factors such as the presence of weak instruments before deciding that the magnitude of any estimator bias reflects specification errors coming from breaking means. 
The next section sets out our simple model and distinguishes three estimators of the NKPC. One of these cannot be implemented in practice but gives a useful benchmark, and it has often appeared in empirical and theoretical work. Section 3 then provides a simplified account of how the NK model can be solved in the presence of structural change. Section 4 looks at a range of simulations, beginning with no breaks, moving on to breaks in the reduced form but not the structure, and finishing with breaks in both the structure and reduced form. Breaks in the reduced form correspond to breaks in the means of the inflation rate etc., while breaks in the structure come from changes in the intercept in the NKPC. The estimators introduced in Section 2 are examined, and we assess which one performs best in the presence of breaks. In this section we also investigate the robustness of our results to agents not knowing precisely the timing of breaks when they form expectations.

Section 5 looks at the empirical work in Castle et al (2011) on the NKPC in the Euro Area and in the U.S. They argue that the structural equation requires the addition of a number of indicator variables which, when added, reduce the estimated expectations coefficient by a large amount. Of course indicators are only very short-lived breaks, whereas the breaks we look at in this paper are rather longer-lived. Nevertheless, even short-lived breaks can cause specification bias, and their presence in the reduced form can lead to weak instruments. We find that the changed estimated expectations coefficients they report do not stem from any structural change but rather come from extra regressors they add into the NKPC i.e. there is a change of specification. There are however weak instrument issues when trying to estimate their revised specification. We attempt to allow for these and, after doing so, conclude that the forward expectation coefficient estimates do seem to be quite high. Section 7 concludes.

\section{The Model and Estimators}

Designating $\pi_{t}, x_{t}$ and $r_{t}$ as the inflation rate, real marginal cost and the interest rate respectively, while $\bar{\pi}_{t}, \bar{x}_{t}$ and $\bar{r}_{t}$ are their long-run equilibrium values $^{1}$, we will be using the system of equations

\footnotetext{
${ }^{1}$ The values to which $\pi_{t}, x_{t}$ and $r_{t}$ would converge in the absence of shocks.
} 


$$
\begin{aligned}
\pi_{t} & =\left\{(1-s) \bar{\pi}_{t}-\delta \bar{x}_{t}\right\}+\alpha \pi_{t-1}+\gamma \mathbb{E}_{t} \pi_{t+1}+\delta x_{t}+\varepsilon_{1 t} \\
x_{t} & =\left\{\left(1-\rho_{1}\right) \bar{x}_{t}-d \times \overline{r r}\right\}+\rho_{1} x_{t-1}+d\left(r_{t-1}-\pi_{t-1}\right)+\varepsilon_{2 t} \\
r_{t} & =\left\{\left(1-\lambda_{1}\right) \bar{r}_{t}-\lambda_{2} \bar{x}_{t}-\lambda_{3} \bar{\pi}_{t}\right\}+\lambda_{1} r_{t-1}+\lambda_{2} x_{t}+\lambda_{3} \pi_{t}+\varepsilon_{3 t}
\end{aligned}
$$

where $\bar{r}_{t}=\overline{r r}+\bar{\pi}_{t}, \overline{r r}$ is the equilibrium real rate of interest and $s=\alpha+\gamma$. The only two equilibrium values that are allowed to change in our experiments are the inflation target $\left(\bar{\pi}_{t}\right)$ and real marginal cost $\left(\bar{x}_{t}\right)^{2}$. We will study cases in which $\bar{\pi}_{t}$ and $\bar{x}_{t}$ take on just two values, thereby capturing breaks in the unconditional means of these variables at some point in the sample. This is in order to study the results in Castle et al (2012) more carefully. There are other specifications of $\bar{\pi}_{t}$ and $\bar{x}_{t}$ that might be employed e.g. one might have them following Markov Switching (MS) processes. However, unless an absorbing state was allowed, this does not produce breaks in the unconditional means, since in standard MS models the unconditional moments are constant. Another specification that has been suggested involves $\bar{\pi}_{t}$ evolving as a unit root process - - see Ascari (2004) and Cogley and Sbordone (2008). With this however inflation would have an infinite variance. Moreover it would have to be that the slope coefficients in the NKPC above would change with $\bar{\pi}_{t}$. Apart from the fact that the existing derivations of the models don't seem to allow for the fact that the linearization is around a unit root process, it would conflict with our assumption that the coefficients in the NK system are constant. So none of these alternatives are really designed to capture breaks in the unconditional moments, which is the central feature of Castle et al (2012). Nevertheless, it should be said that, if $\bar{\pi}_{t}$ evolved as either an MS or an integrated process, and one ignored such a feature when estimating an NK Phillips curve, then clearly the mis-specification would have some effects on the estimated parameters of a model which assumes that $\bar{\pi}$ is constant. But this involves a different type of mis-specification to that proposed in Castle et al, and so we do not deal with it in this paper.

It is necessary to explain how these equations were arrived at. The NK system we use is simplified in a number of ways. Because our conclusion will be that an upward bias in the NK Phillips curve may stem from weak instruments rather than breaks in means, it is desirable to have models in which the instruments are as strong as possible, so as to highlight the impact

\footnotetext{
${ }^{2}$ Of course $\bar{r}_{t}$ changes because $\bar{\pi}_{t}$ changes.
} 
of breaks. In the standard NK model often used for illustrating monetary policy there are terms in (2) such as $r_{t}-\mathbb{E}_{t} \pi_{t+1}$ rather than $r_{t-1}-\pi_{t-1}$. To explain our choice note that because both $x_{t}$ and $\pi_{t-1}$ are present in the Phillips curve, it will be necessary to find another instrument for $\pi_{t+1}$ if one is to use the estimation procedure in Castle et al. Lagged values of $x_{t}$ and $\pi_{t-1}$ can help, but these are often weak instruments. Consequently, because $r_{t-1}$ does not appear in the equation but is present in the system above it is the obvious instrument. But for it to be a good instrument for $\pi_{t+1}$ it must be the case that $\pi_{t+1}$ depends reasonably strongly on interest rates in the past - placing $r_{t-1}$ in the marginal cost equation accomplishes this. This is also a reason for omitting $\mathbb{E}_{t} x_{t+1}$ from both (2) and (3). One could work with other systems but weak instrument problems will be even larger than we find and this distracts attention from the biases coming from breaks. Finally, the system above deliberately makes $x_{t}$ weakly exogenous, so that one does not need an extra instrument for it in the NK Phillips curve. This assumption again strengthens the instrument set.

Generally the NK system comes from a set of equations that have been log linearized around steady state values. Thus the NK Phillips curve generally has the form

$$
\tilde{\pi}_{t}=\alpha \tilde{\pi}_{t-1}+\gamma \mathbb{E}_{t} \tilde{\pi}_{t+1}+\delta \tilde{x}_{t}+\varepsilon_{1 t},
$$

where $\tilde{\pi}_{t}=\pi_{t}-\bar{\pi}$ and $\tilde{x}_{t}=x_{t}-\bar{x}$ are deviations from the steady state values. Now the simple extension of this to a varying equilibria case would seem to be to define $\tilde{\pi}_{t}=\pi_{t}-\bar{\pi}_{t}$ and $\tilde{\pi}_{t \pm 1}=\pi_{t \pm 1}-\bar{\pi}_{t \pm 1}$. This is straightforward for $\tilde{\pi}_{t}$ but less so for $\tilde{\pi}_{t \pm 1}$. Consider the expectation under what would seem to be the straightforward definition of $\mathbb{E}_{t}\left(\tilde{\pi}_{t+1}\right)$ viz. $\mathbb{E}_{t}\left(\tilde{\pi}_{t+1}\right)=\mathbb{E}_{t}\left(\pi_{t+1}-\bar{\pi}_{t+1}\right)$. At the point where expectations are formed agents think the target inflation rate is $\bar{\pi}_{t}$, and it is not until $t+1$ that their beliefs change. So it seems more appropriate to use $\mathbb{E}_{t}\left(\pi_{t+1}-\bar{\pi}_{t}\right)$ rather than $\mathbb{E}_{t}\left(\pi_{t+1}-\bar{\pi}_{t+1}\right)$ to represent $\mathbb{E}_{t}\left(\tilde{\pi}_{t+1}\right)$. In a similar vein, what matters to agents when setting $\pi_{t}$ is how far away the inflation rate at $t-1$ is from the equilibrium at $t$. Consequently $\tilde{\pi}_{t-1}$ is best defined as $\pi_{t-1}-\bar{\pi}_{t}$. Using those definitions produces (1). Similar arguments give (2) and (3).

We can think of three possible IV estimators of the Phillips curve. The first, the unrestricted estimator (UE), works with the following equation, where \{\} contains the error term,

$$
\pi_{t}=b_{1}+\alpha \pi_{t-1}+\gamma \pi_{t+1}+\delta x_{t}+\left\{\gamma\left(\mathbb{E}_{t} \pi_{t+1}-\pi_{t+1}\right)+\varepsilon_{1 t}\right\} .
$$


Variables that are uncorrelated with $\varepsilon_{1 t}$ provide instruments for the regressors and these are $\pi_{t-1}, x_{t}, x_{t-1}$ and $r_{t-1} . x_{t}$ qualifies as an instrument because only lagged values determine it and $\varepsilon_{2 t}$ is uncorrelated with $\varepsilon_{1 t}$. The appendix shows that $\pi_{t+1}$ depends on $x_{t}, \pi_{t-1}$ and $r_{t-1}$ but not $x_{t-1}$. Hence $x_{t-1}$ is not a relevant instrument for $\pi_{t+1}$, a fact noted by Pesaran (1987) when $d$ in (2) is zero. Consequently, $\pi_{t-1}, x_{t}$ and $r_{t-1}$ provide exactly the right number of instruments for the three variables $\pi_{t-1}, \pi_{t+1}$ and $x_{t}$. In much empirical work a broader set of instruments is assumed without specifying a model, but it is useful to have a small model from which to generate the instruments.

A second estimator - the restricted IV estimator (RE) - works with the re-parameterized equation

$$
\pi_{t}-s \pi_{t-1}=b_{1}+\gamma\left(\pi_{t+1}-\pi_{t-1}\right)+\delta x_{t}+\left\{\gamma\left(\mathbb{E}_{t} \pi_{t+1}-\pi_{t+1}\right)+\varepsilon_{1 t}\right\},
$$

where two instruments are needed for $\pi_{t+1}-\pi_{t-1}$ and $x_{t}$. These are found by combining together the three potential ones $-\pi_{t-1}, x_{t}$ and $r_{t-1}$.

Finally, there is a third estimator which applies RE assuming that $s=1$. We call this RES. The rationale for RES comes from Gali and Gertler's (1999) NK model - when the discount rate is .99 the value of $s$ varies between .99 and .996. This variation comes from changing either the probability of firms resetting prices or the fraction of firms setting optimal prices when both are allowed to vary over their permissible ranges of zero to one. The RES estimator will therefore be biased in large samples since the error term will be augmented by $(1-s) \pi_{t-1}$, and that will be correlated with the instruments. There are other estimators e.g. Magnusson and Mavroeidis (2010) look at estimating the same model as used with RES but with a minimum-distance approach. However, the methods above are directly connected with those used by Castle et al (2012), in that they emphasise instrumental variables, and so we restrict our attention to the effects of structural breaks on them.

\section{A Simple Variant of the Solution Algorithm}

Kulish and Pagan (2012) set out the algorithm used to compute solutions to the system. It has more general application than the context we are working with here, so we provide a simplified discussion of its workings in order to highlight some significant features of it.

The system we consider has the format

$$
z_{t}=c_{t}+A z_{t-1}+B \mathbb{E}_{t} z_{t+1}+\varepsilon_{t}
$$


where $z_{t}$ is a vector of $n(=3)$ variables $\left(\pi_{t}, x_{t}, r_{t}\right), c_{t}$ are the intercepts in the equations, and $\varepsilon_{t}$ are i.i.d. shocks with zero means and constant variances. It is necessary to allow agents to have potentially different beliefs about the timing of any breaks than what the reality is. In order to study the effects of mean breaks we assume that agents always know the parameters $A$ and $B$ of the system, and it is only $c_{t}$ that their beliefs may be incorrect about. In most of our experiments agents will be taken as believing that the intercepts of the three equations at time $t$ have the true values $c_{t}$, but in our last experiment they believe the intercepts are $c_{t}^{a}$ rather than $c_{t}$. Thus, in general agents solve the system (6) replacing $c_{t}$ with $c_{t}^{a}$ when forming their expectations $\mathbb{E}_{t}^{a} z_{t+1}$, where the "a" indexes the agent's beliefs. Those expectations will then determine the actual outcomes of the system i.e. the observed variables will be consistent with

$$
z_{t}=c_{t}+A z_{t-1}+B \mathbb{E}_{t}^{a} z_{t+1}+\varepsilon_{t} .
$$

Of course when $c_{t}^{a}=c_{t}, \mathbb{E}_{t} z_{t+1}$ and $\mathbb{E}_{t}^{a} z_{t+1}$ coincide.

We adopt the Binder and Pesaran (1995) solution method for solving the system. Briefly this involves converting (6) into a purely forward looking form, solving that, and then recovering the solution to the original system. Consequently, we first need to compute what agents' expectations are at $t$. At that point they know $z_{t-1}$ so the system will be converted to one involving $Z_{t}^{a}=z_{t}^{a}-P z_{t-1}$. Then agents believe that the system is generated by (7) with $c_{t}=c_{t}^{a}$ and, after performing the conversion, this leaves

$$
Z_{t}^{a}=(I-B P)^{-1} c_{t}^{a}+S \mathbb{E}_{t}^{a}\left(Z_{t+1}^{a}\right)+Q \varepsilon_{t},
$$

where $S=(I-B P)^{-1} B, Q=(I-B P)^{-1} T$ and $P$ is chosen such that $\left(A+B P^{2}-P\right)=0$. Because shocks $\varepsilon_{t}$ are i.i.d. the solution for $Z_{t}^{a}$ will be ${ }^{3}$

$$
Z_{t}^{a}=\sum_{j=0}^{\infty} S^{j}(I-B P)^{-1} c_{t+j}^{a}+Q \varepsilon_{t} .
$$

Thereupon

$$
z_{t}^{a}=P z_{t-1}+\sum_{j=0}^{\infty} S^{j}(I-B P)^{-1} c_{t+j}^{a}+Q \varepsilon_{t},
$$

\footnotetext{
${ }^{3}$ Of course we need the eigenvalues of $S$ to be less than unity.
} 
meaning that

$$
\mathbb{E}_{t}^{a} z_{t+1}=P z_{t}+\sum_{j=1}^{\infty} S^{j}(I-B P)^{-1} c_{t+j}^{a} .
$$

It is interesting to observe here that, when agents form expectations, they do so by weighting the current data $z_{t}$ with what they expect about future developments. Since the realized $z_{t}$ will incorporate any structural change that has happened, agents' expectations will partially adapt to this, even when their fundamental beliefs are incorrect.

Now the realizations of $z_{t}$ will be found by putting the agents' expectations into (7) giving

$$
z_{t}=c_{t}+A z_{t-1}+B\left(P z_{t}+\sum_{j=1}^{\infty} S^{j}(I-B P)^{-1} c_{t+j}^{a}\right)+\varepsilon_{t} .
$$

This form remains valid when $c_{t}^{a}=c_{t}$ i.e. reality and beliefs coincide.

It is worth asking what estimation issues arise for the NK Phillips curve when reality and beliefs do not coincide. The Phillips curve from (4) will be taken to have the form

$$
\tilde{\pi}_{t}=\alpha \tilde{\pi}_{t-1}+\gamma \mathbb{E}_{t}^{a} \tilde{\pi}_{t+1}+\delta \tilde{x}_{t}+\varepsilon_{1 t},
$$

as it is what agents believe that determines expectations. Here $\tilde{\pi}_{t+1}$ was defined as $\pi_{t+1}-\bar{\pi}_{t}$ and so it will be $\pi_{t+1}-\bar{\pi}_{t}^{a}+\bar{\pi}_{t}^{a}-\bar{\pi}_{t}$. Accordingly, any estimator that fails to deal with the divergence between $\bar{\pi}_{t}^{a}$ and $\bar{\pi}_{t}$ will be biassed, as that discrepency will become part of the error term. This situation happens only when $c_{t}^{a} \neq c_{t}$, which occurs with the last of our five experiments in the next section. In all the other experiments $c_{t}=c_{t}^{a}$ i.e. agents know when the break occurs and its magnitude, so that bias in any estimator does not come from agents failing to know about the break, but are simply due to the fact that the econometrician estimating the NK Phillips curve is assuming that there are no breaks. 


\section{Simulation Experiments}

\subsection{Parameter Values}

In the experiments we report on later some parameters in the NK model are assumed constant. These are

$$
\begin{aligned}
\alpha & =(.7, .3), s=.99, \delta=.1, \rho=.7, \bar{\pi}=0, \bar{x}=0 \\
\overline{r r} & =.02, d=-.1, \lambda_{1}=.7, \lambda_{2}=.2, \lambda_{3}=.5 . \\
\sigma_{\varepsilon_{1}} & =.001, \sigma_{\varepsilon_{2}}=.001, \sigma_{\varepsilon_{3}}=.001
\end{aligned}
$$

The parameter values above seem fairly standard. $s$ was chosen to be .99 owing to the fact that in NK Phillips curves $s$ must equal or exceed the discount rate. Two values of $\alpha$ are allowed for to reflect either a weak $(\gamma=$ $.99-\alpha=.29)$ or strong $(\gamma=.69)$ effect from forward looking expectations.

The structural changes we allow for are in either $\bar{\pi}$ or $\bar{x}$. If there are no breaks (experiment 1) these remain zero throughout. In the remaining experiments there is a single break in either the inflation target or real marginal cost. When it is the former $\bar{\pi}$ becomes .02 after forty percent of the sample while, if it is the latter, $\bar{x}$ becomes -.015 at that point in the sample. Experiment 2 is an exception. Here a break in the inflation target occurs as above, but we allow real marginal cost to move from zero to $\bar{x}_{t}=\frac{(1-s) \bar{\pi}_{t}}{\delta}$. This choice means that the intercept in the NK Phillips curve (5), $b_{1}$, is zero i.e. it does not show any breaks. Experiments 3 and 4 then look at changing either the mean inflation rate or the mean of marginal cost. Finally, experiment 5 looks at whether our conclusions depend on agents correctly knowing the timing of any breaks. As pointed out at the end of the previous section this introduces an extra form of mis-specification. To assess the impact of such specification error we consider the case where agents believe that the means $\bar{x}, \bar{\pi}$ remain constant until $60 \%$ of the sample is completed, whereas the actual shift is at $40 \%$ of the sample.

The six experiments were chosen to elucidate many of the issues mentioned in the introduction to the paper. When examining the results our focus will be on two things. Firstly, even if there is no structural change, we can have a small-sample bias in the estimator of $\gamma$, simply because of weak instruments ${ }^{4}$. Secondly, when the intercept does shift, and no allowance is

\footnotetext{
${ }^{4}$ In fact the bias can exist for very large samples, even though it disappears asymptotically.
} 
made for that, a specification error is created, which can cause a large-sample bias. One way to distinguish these two effects is to have breaks in means but none in the NK Phillips curve intercept i.e. they offset as in Experiment 2. Then any bias must be due to weak instruments since the equation is correctly specified.

One way to recognize weak instruments ( infeasible in practice) is to compare the mean and median of the estimators, since in many simulations ( but not all) weak instruments show up as the IV estimator being non-normally distributed. Another way is to extend the sample and see if the bias goes away. Finally, for the UE (RE), examining the $\mathrm{F}$ test for a zero coefficient of $r_{t-1}\left(r_{t-1}, \pi_{t-1}\right)$ in the regression of $\pi_{t+1}\left(\pi_{t+1}-\pi_{t-1}\right)$ against $x_{t}, \pi_{t-1}$ and $r_{t-1}$ will give useful ( feasible) information about weak instruments. The popular rule of thumb that $F>10$ is often used for assessing the quality of the instruments. In interpreting later results it should be noted that structural change not only affects intercepts in equations like the Phillips curve, but can also make instruments stronger or weaker due to changes in the reduced form, and this is discussed in experiment 2. As mentioned earlier breaks occur at $40 \%$ of the sample size and, when agents have the timing wrong, they expect them to have been at $60 \%$. We use percentages of the sample size, as this enables us to increase the sample size so as to study the "asymptotic" properties, as well as the small sample ones.

\subsection{The Experiments}

\subsubsection{Experiment 1: No breaks}

This experiment keeps all parameters constant and $\bar{x}, \bar{\pi}$ are both set to zero. Two sample sizes are used, $T=100$ and $T=1000$, and 500 replications are performed to assess estimator bias. The estimators are the unrestricted (UE) and two restricted estimators - one of these uses the correct sum of the forward and backward parameters (RE) and the other just sets it to unity (RES). Table 1 contains the results. 


\begin{tabular}{|c|c|c|c|c|c|c|c|}
\hline $\mathrm{T}=100$ & \multicolumn{3}{|c|}{ True $\gamma=.29$} & \multicolumn{4}{|c|}{ True $\gamma=.69$} \\
\hline & UE & $\mathrm{RE}$ & RES & $\mathrm{UE}$ & $\mathrm{RE}$ & RES & \\
\hline Median & .48 & .42 & .48 & .67 & .68 & .69 & \\
\hline Mean & -.95 & .39 & .46 & 2.09 & .69 & .70 & \\
\hline $\mathrm{T}=1000$ & & & & & & & \\
\hline Median & .42 & .31 & .44 & .68 & .69 & .70 & \\
\hline Mean & .27 & .31 & .43 & .70 & .69 & .70 & \\
\hline & & & & & & & \\
\hline
\end{tabular}

A weak instrument bias in the UE shows up strongly here. In the case of weak forward looking expectations $(\gamma=.29)$ it is still present when there are 1000 observations. The $\mathrm{F}$ tests for instrument quality in the UE case are $.72(T=100)$ and $10.6(T=1000)$. There are two free instruments for the $\mathrm{RE}$ and these are much stronger for the variable that needs instrumenting in the RES case $-\pi_{t+1}-\pi_{t-1}-$ with the F statistics being $36.4(T=100)$ and $457(T=1000)^{5}$.

It is clear that the performance of the RE detoriates as $\gamma$ declines. The reason is that inflation becomes more persistent and, since we are effectively looking at the correlation of $\pi_{t+1}-\pi_{t-1}$ with the instrument $\pi_{t-1}$, this declines with $\gamma$. The RES estimator not only has a weak instrument bias but one due to the invalid assumption that $\gamma+\alpha=1$. For small $\gamma$ this can be sizable, as the $T=1000$ case shows. If there is actually a weak effect of forward expectations then the RES estimator will suggest that it is stronger than it really is. However, if the expectations effect is strong then the RE and RES biases are quite small. In practice many people have imposed the restriction that $s=1$ in estimation. It should be noted however that the bias could be lower than shown here as we have replaced the true $s=.99$ with an assumed value of $s=1$, whereas a discount factor of .99 would imply that the true value of $s$ lies much closer to unity than we have assumed in this experiment. Since the bias depends directly on the difference between the assumed and true values it can be much smaller if these are close.

\footnotetext{
${ }^{5}$ These are generally averages of the $F$ tests for weak instruments across all replications, although when $T=1000$ they would be close to the point estimate using just the 1000 observations.
} 


\subsubsection{Experiment 2: Breaks in Means but not in the Intercept of the Phillips Curve}

Here we allow $\bar{\pi}_{t}$ to break, going from zero to .02, but choose $\bar{x}_{t}$ so that the intercept in the Phillips curve remains constant. All other parameters are as in Experiment 1. As the standard deviation of inflation is .003 this is an enormous break and is chosen to maximize the effects. There is no specification bias in the UE and RE estimators due to the compensating variation in $\bar{x}_{t}$, so that any biases in UE and RE must come solely from weak instruments.

Table 2 shows that the UE estimator behaves in much the same way as it did before, although it may be slightly better behaved. Using the F test the "free" instrument $r_{t-1}$ does seem of higher quality. This happens due to the break in the inflation process. The RE performance has weakened because the break in the inflation process has made the latter much more persistent, and so $\pi_{t-1}$ is a weaker instument for $\pi_{t+1}-\pi_{t-1}$. This demonstrates an interesting feature: structural breaks may affect the quality of instruments. Indeed they might even become better. To see why suppose we assume that $\pi_{t}=\varepsilon_{t}$ was $i . i . d(0,1)$. Then, if we regressed $\pi_{t+1}$ on $\pi_{t-1}$, in large samples we would find a regression coefficient of zero. This is also the correlation coefficient since the standard deviation of $\pi_{t}$ is unity. Now consider what happens if $\pi_{t}=\varepsilon_{t}$ for $t=1, \ldots, T_{1}$ and $\pi_{t}=\varepsilon_{t}+\mu$ for $t=T_{1}+1, \ldots, T$, where $T_{1}=\frac{T}{2}$. Then the regression coefficient will be

$$
\begin{aligned}
\frac{\frac{1}{T} \sum_{t=1}^{T} \pi_{t-1} \pi_{t+1}}{\frac{1}{T} \sum_{t=1}^{T} \pi_{t-1}^{2}} & =\frac{.5 \frac{1}{T_{1}} \sum_{t=1}^{T_{1}} \pi_{t-1} \pi_{t+1}+.5 \frac{1}{T_{1}} \sum_{t=T_{1}+1}^{T} \pi_{t-1} \pi_{t+1}}{.5 \frac{1}{T_{1}} \sum_{t=1}^{T_{1}} \pi_{t-1}^{2}+.5 \frac{1}{T_{1}} \sum_{t=T_{1}+1}^{T} \pi_{t-1}^{2}} \\
& \longrightarrow \frac{\mu^{2}}{\left(2+\mu^{2}\right)}
\end{aligned}
$$

so the shift raises the regression coefficient. In the case where $\mu=1$ the correlation of $\pi_{t+1}$ and $\pi_{t-1}$ is now .24 and, when $\mu=2$, it rises to .53. Thus structural change can improve the quality of the instrument. It looks as if the RES performs much the same but the bias increases as the sample size rises. When $T=5000$ the mean/median of of $\hat{\gamma}_{R E S}=.9$, so that the upward bias in $\hat{\gamma}$ due to assuming $\gamma+\alpha=1$ seems to be between .15 and .2 . 


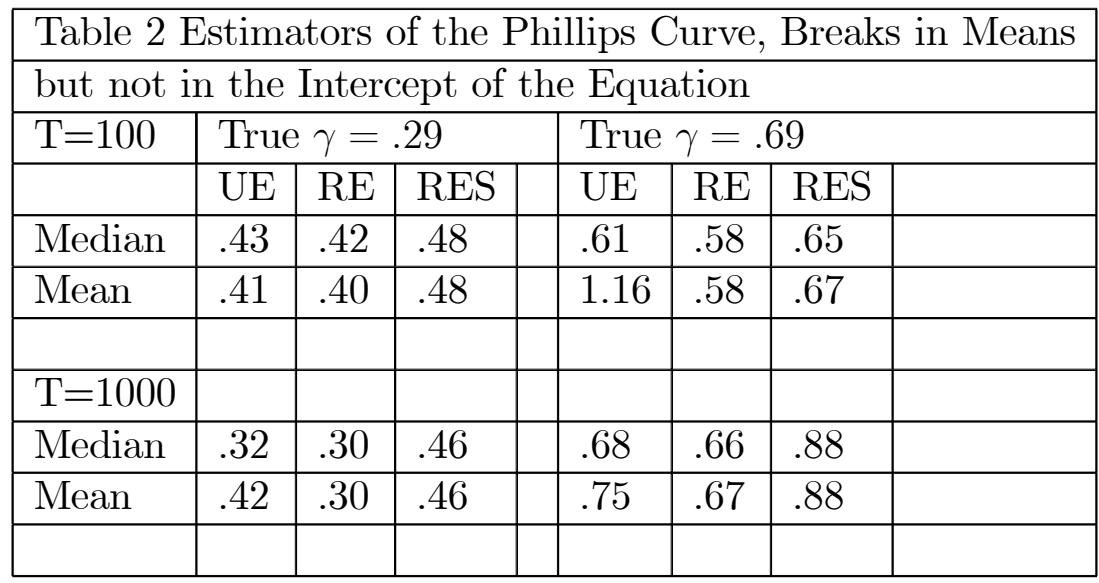

\subsubsection{Experiment $3 \pi$ Breaks, Intercept in Phillips Curve Shifts}

The experiment now eliminates the offsetting change in $\bar{x}$ of the preceding experiment, resulting in $\bar{x}$ not changing. It should be noted that, even though a large break in $\bar{\pi}$ is allowed ( it equals five standard deviations ), this does not lead to a very large break in the Phillips curve intercept, as the latter is $(1-s) \bar{\pi}_{t}$. Table 3 gives the results.

\begin{tabular}{|l|c|c|c|c|c|c|c|c|}
\hline \multicolumn{10}{|c|}{ Table 3: Estimators of the Phillips Curve, Break in Inflation Mean } \\
\hline \multicolumn{10}{|c|}{ T=100 } & True $\gamma=.29$ & \multicolumn{3}{|c|}{ True $\gamma=.69$} \\
\hline & UE & RE & RES & & UE & RE & RES & \\
\hline Median & .43 & .38 & .46 & & .61 & .43 & .54 & \\
\hline Mean & .35 & .37 & .44 & & .61 & .42 & .54 & \\
\hline & & & & & & & & \\
\hline T=1000 & & & & & & & & \\
\hline Median & .41 & .24 & .42 & & .70 & .32 & .66 & \\
\hline Mean & .39 & .21 & .42 & & 1.49 & .33 & .66 & \\
\hline & & & & & & & & \\
\hline
\end{tabular}

The instruments for the UE are now better than for the RE ( at $T=1000$ the $\mathrm{F}$ test is 9 versus 6.5 ) when $\gamma=.29$ and, although the instruments are rather weak, the UE results mainly reflect the specification error bias due to the structural change. This is seen by noting that, at $T=10000$, the mean/median of $\hat{\gamma}_{U E}=.4$. UE instruments deteriorate when $\gamma=.69$ and this shows up as slow convergence to a normal density. At $T=10000$ the 
mean/median of $\hat{\gamma}_{U E}$ is .72 ( and the mean and median are the same), so the bias due to specification error is actually quite small when there is a strong effect from expectations. The RE ( and RES) case is also an amalgam of weak instrument and specification error bias. At $T=10000$ the mean/median of $\hat{\gamma}_{R E}$ are . $2(\gamma=.29)$ and $.4(\gamma=.69)$, so that the weak instrument bias in smaller samples is apparent. Notice that, in this case, the RE is biaseddownwards due to the structural change, and this is also true of RES, showing that breaks in the mean of inflation cannot always be assumed to lead to an upward bias.

It is worth looking at what would happen if the structural change had been earlier in the sample. So we set it at $20 \%$ of the sample. As expected there are changes in the magnitudes but the patterns are much the same. Thus when $\gamma=.69$ and $T=1000$ we find the medians of the $U E$ and $R E$ to now be .67 and .42 respectively, versus the .70 and .32 of Table 3 . When $T=100$ the corresponding results are .59 and .45 versus .61 and .42 . So our results seem robust to the break occuring early in the sample. Making it occur at $80 \%$ of the sample produces the same type of outcomes. Generally the results in both cases are better for all estimators simply because there is a larger fraction of the sample where the model has no breaks. Of course in the limit where all the sample reflects no breaks then we would be simply looking at biases that come from weak instruments. By setting it at $40 \%$ of the sample we are allowing structural change to potentially have a significant effect.

\subsubsection{Experiment $4 x$ Breaks, Intercept in Phillips Curve Shifts}

The size of the break in $\bar{x}$ is -.015. Again this is some five times the standard deviation and so is very large. Because it is not multiplied by (1-s) in the Phillips curve intercept ( as was $\bar{\pi}$ ) it produces large changes in the latter. Instruments are quite good in this case for UE when $\gamma=.29$, although much better for the RE estimator. When $\gamma=.69$ the UE instrument has an F test of 11.2 when $T=1000$ while the $\mathrm{RE}$ value is 16.2 . This shows up in the relative performances. A sample of $T=1000$ gives estimates that are only a little different from larger samples. Consequently, while there is an upward bias for all estimators ( due to structural change) when $\gamma=.29$, there is a downward bias when $\gamma=.69$. Again the bias is relatively small if there are strong expectations effects. 


\begin{tabular}{|l|c|c|c|c|c|c|c|c|}
\hline \multicolumn{6}{|l|}{ Table 4: Estimators of the Phillips Curve, } \\
\hline Breaks in Mean of Marginal Cost \\
\hline T=100 & \multicolumn{1}{l|}{ True $\gamma=.29$} & \multicolumn{4}{l|}{ True $\gamma=.69$} \\
\hline & UE & RE & RES & UE & RE & RES & \\
\hline Median & .51 & .44 & .48 & .57 & .63 & .64 & \\
\hline Mean & .61 & .43 & .48 & .40 & .63 & .64 & \\
\hline & & & & & & & & \\
\hline T=1000 & & & & & & & & \\
\hline Median & .51 & .41 & .49 & & .65 & .65 & .67 & \\
\hline Mean & .52 & .41 & .49 & & .67 & .65 & .67 & \\
\hline & & & & & & & & \\
\hline
\end{tabular}

\subsubsection{Experiment 5 - Experiment 3 but Agents think Breaks at $60 \%$ of Sample}

By comparing Tables 3 and 5 it is clear that the properties of the estimators are affected by agents mis-timing the structural change but not by a major amount. It seems as if the UE is more affected than RE and RES.

\begin{tabular}{|l|l|l|l|l|l|l|l|l|}
\hline \multicolumn{10}{|c|}{ Table 5 Estimators of Phillips Curve, Agents Mis-time Break } \\
\hline \multicolumn{10}{|c|}{ T=100 } & True $\gamma=.29$ & \multicolumn{3}{|c|}{ True $\gamma=.69$} \\
\hline & UE & RE & RES & & UE & RE & RES & \\
\hline Median & .43 & .37 & .46 & .39 & .38 & .54 & \\
\hline Mean & -.11 & .37 & .45 & .49 & .37 & .54 & \\
\hline & & & & & & & & \\
\hline T=1000 & & & & & & & & \\
\hline Median & .39 & .19 & .42 & & 1.32 & .43 & .76 & \\
\hline Mean & .41 & .16 & .42 & 3.33 & .43 & .76 & \\
\hline & & & & & & & & \\
\hline
\end{tabular}

All these results seem to suggest that, provided one can find good instruments, the specification bias due to structural change would not be that large when expectations are very important and, even when they aren't, one wouldn't find too big a bias. The RES estimator looks attractive if good instruments are available. 


\section{Breaks and the Euro Area New Keynesian Phillips Curve}

Castle et al. (2011) use the Euro Area NK Phillips curve to illustrate the effects of breaks, arguing that the large coefficient on forward expectations is due to them. The analysis of the preceding section has shown that this could be true, but it was also found that biases in the estimators may come from weak instruments. Consequently, we re-do their analysis here, asking whether structural change is likely to be the cause of any large expectations coefficient estimate.

First, we estimate the hybrid NK Phillips curve over the period 1972/2 to $1998 / 1^{6}$

$$
\pi_{t}=c+\alpha \pi_{t-1}+\gamma E_{t} \pi_{t+1}+\delta x_{t}+\varepsilon_{t},
$$

where $x_{t}$ is real marginal costs ( which is labour share, $s_{t}$, in Castle et al.'s equations). Their paper states that the instruments used were $\left\{\pi_{t-j}\right\}_{j=1}^{5}, x_{t-1}, x_{t-2}$, $g a p_{t-1}, g_{a p_{t-2}}$, where $g a p_{t-1}$ is the Euro Area output gap. Their estimates of (8) are in (9) ( with t ratios in parentheses).

$$
\begin{gathered}
\pi_{t}=.009+.28 \pi_{t-1}+.66 E \pi_{t+1}+.01 x_{t}+\varepsilon_{t} \\
(.8)(2.4)
\end{gathered}
$$

They then suggest that some dummy variables be added on to (9) for 1973/1, 1976/2, 1976/3, 1978/4 and 1983/1. Doing that produces

$$
\begin{gathered}
\pi_{t}=.01+.28 \pi_{t-1}+.65 E \pi_{t+1}+.01 x_{t}+\text { dummies }+\varepsilon_{t}, \\
(.9)(2.6) \quad(5.2)
\end{gathered}
$$

so that the parameter estimates have scarcely changed with the introduction of the dummy variables.

How then do they generate a changed coefficient for the forward expectation term? The answer is through an additional regressor - the lagged output

\footnotetext{
${ }^{6}$ We are grateful to Ragner Nymoen for providing the data for both the Euro Area and the U.S. and corresponding with us over its use.
} 
gap - whereupon they get $\hat{\gamma}=.018^{7}$. One might argue that the addition of the output gap enables the mark-up to vary with the state of demand, and so the new specification makes sense. But the changed coefficient on expectations comes from the new specification, not from any structural change, if that is what the dummy variables represent. In fact, regressing $\pi_{t+1}$ on the dummies either by themselves, or in the presence of other regressors such as $\pi_{t-1}$ and $g_{a p_{t-1}}$, produces very low $\mathrm{F}$ tests for the hypothesis that these have any relationship with $\pi_{t+1}$. But this is supposed to be the mechanism whereby structural change affects the estimated expectations coefficient. To make this point more forcibly consider experiment 3 . Taking $\mathrm{T}=100$ we regress $\pi_{t+1}$ on a dummy that equals zero for $40 \%$ of the sample and unity for the remainder. The $\mathrm{F}$ test that the dummy has no impact is 15 . One problem is that the dummy variables used in the Euro Area NK Phillips curve seem to be more "blips" than breaks, as they are not sustained, and so have little effect on the expectations coefficient.

We might ask whether imposing the "adding-up" constraint that $\gamma+\alpha=1$ makes a difference? Doing so we find that $\hat{\gamma}_{R E S}=.72$. It should be admitted that the downside for RES here is that neither $x_{t}$ nor $\operatorname{gap}_{t-1}$ are significant, and they have negative estimated coefficients, although if one took a Bayesian perspective and insisted that they be positive, this would not be inconsistent with the data. Nevertheless, it has to be said that the main reason for imposing $\gamma+\alpha=1$ was its potential to improve the quality of instruments a lot, and this was certainly the case in many of the experiments. But, with the Euro Area data set, instruments are extremely weak for both the UE and RES estimators. It is easy to understand why: inflation is very persistent, with an $A R(1)$ coefficient of .98. Consequently, $\pi_{t-1}$ is by far the biggest contributor to the explanation of $\pi_{t+1}$. When applying the UE $\pi_{t-1}$ is effectively not available to instrument $\pi_{t+1}$ and so the best instrument is excluded. For the $\mathrm{RE} \pi_{t-1}$ is certainly available as an instrument for $\left(\pi_{t+1}-\pi_{t-1}\right)$ but the high persistence means it is a weak instrument. So, even though one can say any bias cannot be due to structural change, it may be that there is a high upward bias coming from weak instruments.

At the end of their paper Castle et al. look at the NK Phillips curve estimated over a period that they think has no breaks - 1983/2-1998/1. The idea is to see if one would get a high estimate for the forward expectation

\footnotetext{
${ }^{7}$ This is sensitive to the instruments used. They decided not to use lagged wage growth as instruments but, if one does, the coefficient becomes .27 .
} 
coefficient during this era. They report that $\hat{\gamma}_{U E}$ is now .082 and so conclude ".. confirming that its significance ...is as a proxy for unmodeled shifts" i.e. the high value found in the earlier era was due to breaks. It is interesting to note that, if one added $\Delta w_{t-1}$ and $\Delta w_{t-2}$ ( lagged wage inflation) into the instrument set, rather than excluding it as Castle et al do, then $\hat{\gamma}_{U E}$ would be .61. Moreover, $\hat{\gamma}_{R E S}=.70$. So, just as for the "breaks" sample, there is a conflict between the different estimators and we seek to resolve it.

Now for the RES to be trustworthy low persistence in inflation is needed. The post 1983/1 sample satisfies this quite well, as the $\operatorname{AR}(1)$ coefficient is .83. So we would expect that $\pi_{t-1}$ would be a reasonable instrument for $\pi_{t+1}-\pi_{t-1}$. But other instruments are being used and some of these seem to be ineffective, specifically $\pi_{t-3}, . ., \pi_{t-5}$. These contribute nothing to the explanation of $\pi_{t+1}$ ( or even $x_{t}$ ). It is not a good policy to utilize weak instruments that are superfluous. So we reduced the instrument set to $\left\{\pi_{t-j}\right\}_{j=1}^{2}, x_{t-1}, x_{t-2}$, gap $_{t-1}$, gap $_{t-2}$.

A second issue is that an instrument for $x_{t}$ is needed as well as one for $\pi_{t+1}$. Regressing $x_{t}$ against the instrument set above shows that the dominant explanator (with a t ratio of 5) is $x_{t-1}$. So, if we think of using $x_{t-1}$ as the instrument for $x_{t}$, this leaves the effective instrument set as $\left\{\pi_{t-j}\right\}_{j=1}^{2}, x_{t-1}, x_{t-2}$, $g a p_{t-2}$ for RES, and the same for UE, but with $\pi_{t-1}$ deleted ( as $\pi_{t-1}$ is a regressor it is effectively not available as an instrument for either $\pi_{t+1}$ or $x_{t}$ ). Testing if the RES instruments contribute to $\pi_{t+1}-\pi_{t}$ we get an $\mathrm{F}$ test of 15 , so the instruments look satisfactory. In contrast the UE instruments have virtually no association with $\pi_{t+1}$, with the $\mathrm{F}$ test being 1.3 . Hence the UE will suffer from weak instruments while the RES will be much less affected.

Given this result it is worth getting the RES for the second era using the Castle et al. instrument set, but excluding $\left\{\pi_{t-j}\right\}_{j=3}^{5}$. If we use the sample from 1983/2 then we get an estimate of the coefficient of $\left(\pi_{t+1}-\pi_{t-1}\right)$ of .74. Because we are using two lags ov some variables as instruments it is probably better to start the sample in $1983 / 4$, as that is now clear of the 1983/1 observation that Castles et al use an impulse indicator for. As seen in (11) this produces a lower estimate of the coefficient of $\left(\pi_{t+1}-\pi_{t-1}\right)$.

$$
\pi_{t}-\pi_{t-1}=.002+.65\left(\pi_{t+1}-\pi_{t-1}\right)+.003 x_{t}-.000001 \operatorname{gap}_{t-1}+\varepsilon_{t} .(11)
$$

There is serial correlation in the specification however, and that may reflect some mis-specification. Computing Newey-West HAC standard errors 


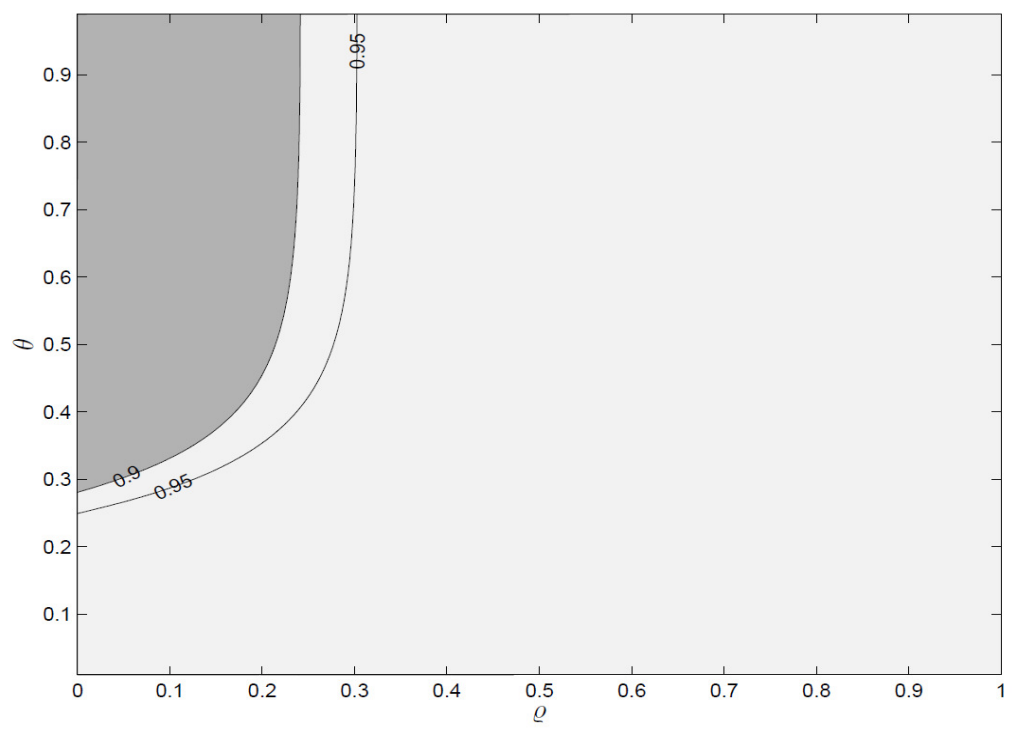

Figure 1: GMM-AR Confidence Sets for the Parameters $\theta$ and $\rho$ of the European NKPC

one finds that the $t$ ratios are only slightly changed. Because of the serial correlation we might do a GMM estimator and this produces an estimator of the expectation coefficient of .68 with a much higher t ratio of 9 , while that for the gap is lower at .7. This leads us to eliminate the gap variable as a regressor and its lags as instruments, and the estimated coefficient of $\left(\pi_{t+1}-\pi_{t-1}\right)$ becomes .69 . This last model now exactly fits the structure used by Magnusson and Mavroeidis (2010) to compute the Anderson-Rubin joint confidence intervals for $\rho$ and $\theta$ in the NK Phillips curve, where the coeffcient of $\pi_{t+1}$ is $\frac{1}{1+\rho}$ and $\theta$ is related to the coefficient of the $x_{t}$ variable. $^{8}$ These are given in Figure 1 and show that the forward expectation coefficient $\left(\frac{1}{1+\rho}\right)$ is at least .6. Of course the sample size here is small, and so the result does need to be treated with quite a deal of caution, but it certainly does not provide evidence against significant forward looking expectation effects upon inflation.

\footnotetext{
${ }^{8}$ We would like to thank Sophocles Mavroides for making the MATLAB program used in Magnusson and Mavroeidis (2010) available to us to perform this computation.
} 
In this last exercise we are using a sample that Castle et al maintain has no structural breaks so that any bias in the forward expectation coefficient would have to come from weak instruments. But the evidence based on robust standard errors suggests that the coefficient remains high. This does not rule out the possibility that there are other specification errors that could have resulted in a high coefficient estimate, but our focus has been upon whether the specification error is that of breaks in the means of variables.

\section{Breaks and the U.S. New Keynesian Phillips Curve}

Castle et al also report on an investigation into how the forward expectations coefficient for the U.S. NKPC changes in the presence of structural breaks. We will look at their investigation of the NKPC over the sample period used by Gali and Gertler (1999), as that shows the weakest effect of forward looking expectations. Estimating the basic NKPC with instruments $\left\{\Delta p_{t-j}\right\}_{j=1}^{4},\left\{\Delta w_{t-j}\right\}_{j=1}^{2},\left\{g a p_{t-j}\right\}_{j=1}^{2}$ and $\left\{x_{t-j}\right\}_{j=1}^{2}$, they find a forward coefficient estimate of $\hat{\gamma}_{U E}=.623$. Augmenting the NKPC with nine dummy variables to capture the structural changes as well as two extra regressors, $\Delta p_{t-3}$ and $g a p_{t-1}$, the estimate then becomes $\hat{\gamma}_{U E}=.253$. Just as for the Euro Area study we therefore ask whether this change was due to the dummy variables or the added regressors? Just putting in the dummy variables we find that $\hat{\gamma}_{U E}=.56$, so the large change is due to the new specification i.e. the added regressors and not to any structural change.

Looking now at the period of time that they feel has no structural change - post 1981/3 - Castle et al fitted their preferred model i.e. with the extra regressors, and found that $\hat{\gamma}_{U E}=.28$. However the gap $_{t-1}$ variable only has a $t$ ratio of 1.3 ( 2.3 for $\Delta p_{t-3}$ ). If this is dropped then $\hat{\gamma}_{U E}=.56$, although $x_{t}$ has a small negative coefficient ( but not different from zero). There is evidence of negative serial correlation in the residuals, which was also present in the form that Castle et al ended up with.

As was true of the Euro Area case many of the instruments do not seem to be useful. Regressions of $\Delta p_{t+1}$ and $x_{t}$ against the complete set of instruments suggests that the only ones that are useful for $\Delta p_{t+1}$ are $\Delta p_{t-2}$ and $\operatorname{gap}_{t-1}$, while $x_{t-1}$ is the only one affecting $x_{t}$. In fact gap $p_{t-1}$ is a very important instrument for $\Delta p_{t+1}$, with a correlation of .21 and a t ratio that 
is close to 4 when $\Delta p_{t+1}$ is regressed against it and the other instruments. We noted above that it didn't seem significant in the stable period, so dropping it from the model enables it to be used as an instrument. Then fitting the model with regressors $\Delta p_{t+1}, \Delta p_{t-1}, \Delta p_{t-3}$ and $x_{t}$ and instruments $\Delta p_{t-1}, \Delta p_{t-2}, \Delta p_{t-3}, x_{t-1}$ and $g a p_{t-1}$, we get $\hat{\gamma}_{U E}=.48$. If we imposed the adding up constaint then $\hat{\gamma}_{U E}=.77$. In this case, the instruments are of course much better, since now $\Delta p_{t-1}$ is available. We can also put $g a p_{t-1}$ into the regression, and it turns out to be insignificant. So the overall evidence in the stable period does seem to suggest a forward expectation coefficient that is around $.5 .^{9}$ In any case the drop in the estimated forward coeffcieint noted by Castle et al is not due to any structural change but rather the re-specification of the NKPC with additional regressors.

\section{Conclusion}

Structural change has been conjectured to lead to an upward bias in the estimated forward expectations coefficient in New Keynesian Phillips curves. We have presented a simple New Keynesian model that enables us to assess this proposition. The model enables us to distinguish the effects of specification error caused by structural change from small sample biases that simply arise due to weak instruments. Experiments suggest that the latter dominates the former.

Imposing the restriction that the forward and backward coefficients sum to unity seems a useful thing to do, as it generally produces better instruments at the expense of a small specification bias. Interesting findings are that biases are relatively small when the forward coefficient is high and that structural change can actually improve the quality of instruments, so it may actually be beneficial.

We looked at empirical studies of the Euro Area and U.S. Phillips curves due to Castle et al. (2011), who concluded that the large expectations coefficient typically found in estimation of these was due to structural change. Our analysis suggests that this is not true. It may be that the large coefficient reflects some mis-specification, but it is not due to structural change. We did find that, even using sample periods where structural change was absent,

\footnotetext{
${ }^{9}$ Magnusson and Mavroeidis (2010) look at an NK Phillips curve without $\Delta p_{t-3}, \Delta w_{t-3}$ and gap $_{t-1}$ over a ( longer) sample period of 1984-2008. They perform inference that is robust to weak instruments and find that $\gamma$ does seem to be around .6-.7.
} 
it could be hard to get a precise estimate for the forward expectations coefficient due to weak instruments. We made some attempts to produce better instruments and to make inferences robust to weak instruments. After doing so it did appear that the forward expectations coefficient estimate was quite high rather than the low values Castle et al (2012) report.

\section{References}

Ascari, G. (2004), "Staggered Prices and Trend Inflation: Some Nuisances", Review of Economic Dynamics, 7, 642-667.

Castle, J.L. J.A. Doornik, D.F. Hendry and R. Nymoen (2012), "Testing the Invariance of Expectations Models of Inflation", Econometric Reviews (forthcoming)

Cogley, T. and A.M. Sbordone (2008), "Trend Inflation, Indexation and Inflation Persistence in the New Keynesian Phillips Curve", American Economic Review, 98, 2101-26.

Galí, J. and M. Gertler, 1999, "Inflation Dynamics: A Structural Econometric Approach," Journal of Monetary Economics, 44, October, 195-222.

Kulish, M. and A.R. Pagan (2012), "Estimation and Solution of Models with Expectations and Structural Changes" (mimeo, Reserve Bank of Australia)

Magnusson, L. and S. Mavroeidis (2010), "Identification-Robust Minimum Distance Estimation of the New Keynesian Phillips Curve", Journal of Money, Credit and Banking, 42, 465-481.

Mavroeidis, S. (2004), "Weak Identification of Forward-looking Models in Monetary Economics", Oxford Bulletin of Economics and Statistics, 66, 609-635.

Pesaran, M.H. (1987), The Limits to Rational Expectations, Basil Blackwell

Russell, B., Banerjee, A., Malki, I., and Ponomareva, N. (2010), "A multiple break panel approach to estimating United States Phillips curves", Unpublished paper, Economic Studies, University of Dundee, Dundee, UK.

\section{Appendix}

The system used is (setting means to zero) 


$$
\begin{aligned}
\pi_{t} & =\alpha \pi_{t-1}+\gamma E_{t} \pi_{t+1}+\delta x_{t}+\varepsilon_{1 t} \\
x_{t} & =\rho_{1} x_{t-1}+d\left(r_{t-1}-\pi_{t-1}\right)+\varepsilon_{2 t} \\
r_{t} & =\lambda_{1} r_{t-1}+\lambda_{2} x_{t}+\lambda_{3} \pi_{t}+\varepsilon_{3 t} .
\end{aligned}
$$

where the shocks $\varepsilon_{j t}$ are uncorrelated with one another and not autocorrelated. It is known that the solution to this system would have the form (for inflation and marginal costs)

$$
\begin{aligned}
& \pi_{t}=\phi_{1} \pi_{t-1}+\phi_{2} x_{t-1}+\phi_{3} r_{t-1}+v_{1 t} \\
& x_{t}=\phi_{4} \pi_{t-1}+\phi_{5} x_{t-1}+\phi_{6} r_{t-1}+v_{2 t} .
\end{aligned}
$$

Substituting (15) into (12) and taking the expectation we get

$$
\pi_{t}=\alpha \pi_{t-1}+\gamma\left(\phi_{1} \pi_{t}+\phi_{2} x_{t}+\phi_{3} r_{t}\right)+\delta x_{t}+\varepsilon_{1 t} .
$$

Gathering terms in (17) produces

$$
\pi_{t}=\psi_{1} \pi_{t-1}+\psi_{2} x_{t}+\psi_{3} r_{t}+v_{t}
$$

where $\psi_{1}=\frac{\alpha}{1-\gamma \phi_{1}}, \psi_{2}=\frac{\gamma \phi_{2}+\delta}{1-\gamma \phi_{1}}, \psi_{3}=\frac{\gamma \phi_{3}}{1-\gamma \phi_{1}}, v_{t}=\frac{\varepsilon_{1 t}}{1-\gamma \phi_{1}}$. Substituting for $r_{t}$ from (14) we get

$$
\begin{aligned}
\pi_{t} & =\psi_{1} \pi_{t-1}+\psi_{2} x_{t}+\psi_{3}\left(\lambda_{1} r_{t-1}+\lambda_{2} x_{t}+\lambda_{3} \pi_{t}+\varepsilon_{3 t}\right)+v_{t} \\
& =a_{1} \pi_{t-1}+a_{2} x_{t}+a_{3} r_{t-1}+\eta_{t}
\end{aligned}
$$

where $a_{1}=\frac{\psi_{1}}{1-\psi_{3} \lambda_{3}}, a_{2}=\frac{\psi_{2}+\psi_{3} d_{2}}{1-\psi_{3} \lambda_{3}}, a_{3}=\frac{\psi_{3} \lambda_{3}}{1-\psi_{3} \lambda_{3}}, \eta_{t}=\frac{v_{t}+\psi_{3} \varepsilon_{3 t}}{1-\psi_{3} \lambda_{3}}$.

Leading $\pi_{t}$ from (19) and substituting for $x_{t+1}$ from (16) gives

$$
\pi_{t+1}=a_{1} \pi_{t}+a_{2} x_{t+1}+a_{3} r_{t}+\eta_{t+1} .
$$

Substituting from (16) again we get

$$
\begin{aligned}
\pi_{t+1}= & a_{1} \pi_{t}+a_{2}\left(\phi_{5} x_{t}+\phi_{4} \pi_{t}+\phi_{6} r_{t}+v_{2 t+1}\right) \\
& +a_{3} r_{t}+\eta_{t+1} \\
= & b_{1} \pi_{t}+b_{2} x_{t}+b_{3} r_{t}+\xi_{t+1}
\end{aligned}
$$


where $b_{1}=a_{1}+a_{2} \phi_{4}, b_{2}=a_{2} \phi_{5}, b_{3}=a_{3}+a_{2} \phi_{6}, \xi_{t+1}=\eta_{t+1}+a_{2} v_{2 t+1}$. Now using (14) for $r_{t}$ in (20) produces

$$
\begin{aligned}
\pi_{t+1} & =b_{1} \pi_{t}+b_{2} x_{t}+b_{3}\left(\lambda_{1} r_{t-1}+\lambda_{2} x_{t}+\lambda_{3} \pi_{t}+\varepsilon_{3 t}\right)+\xi_{t+1} \\
& =d_{1} \pi_{t}+d_{2} x_{t}+d_{3} r_{t-1}+\zeta_{t+1}
\end{aligned}
$$

where $d_{1}=b_{1}+b_{3} \lambda_{3}, d_{2}=b_{2}+b_{3} \lambda_{2}, d_{3}=b_{3} \lambda_{1}, \zeta_{t+1}=\xi_{t+1}+b_{3} \varepsilon_{3 t}$. Finally, replacing $\pi_{t}$ by (19) gives

$$
\begin{aligned}
\pi_{t+1} & =d_{1}\left(a_{1} \pi_{t-1}+a_{2} x_{t}+a_{3} r_{t-1}+\eta_{t}\right)+d_{2} x_{t}+d_{3} r_{t-1}+\zeta_{t+1} \\
& =f_{1} \pi_{t-1}+f_{2} x_{t}+f_{3} r_{t-1}+e_{t+1}
\end{aligned}
$$

where $f_{1}=d_{1} a_{1}, f_{2}=d_{1} a_{2}+d_{2}, f_{3}=d_{1} a_{3}+d_{3}$ and $e_{t+1}=d_{1} \eta_{t}+\zeta_{t+1}$. Hence $\pi_{t+1}$ does not depend on $x_{t-1}$ and so the latter is not a relevant instrument i.e. once we use $x_{t}, r_{t-1}, \pi_{t-1}$ as instruments, $x_{t-1}$ provides no further explanation of the variable we want an instrument for, namely $\pi_{t+1}$. 